\title{
Hidden Phase-Retrieved Fluorescence Tomography
}

\author{
Daniele Ancora ${ }^{1 *}$, Diego Di Battista ${ }^{2}$, Asier Marcos Vidal ${ }^{3}$, Stella Avtzi ${ }^{2,4}$, Giannis \\ ZACHARAKIS $^{2}$, AND ANDREA BASSI ${ }^{1,5}$ \\ ${ }^{1}$ Dipartimento di Fisica, Politecnico di Milano, Piazza Leonardo da Vinci 32, 20133 Milano, Italy \\ ${ }^{2}$ Institute of Electronic Structure and Laser, Foundation for Research and Technology-Hellas, N. Plastira 100, 70013 Heraklion, Greece \\ ${ }^{3}$ Department of Bioengineering and Aerospace Engineering, Universidad Carlos III de Madrid, 28911 Leganés, Spain \\ ${ }^{4}$ Institut de Ciències Fotòniques, Barcelona Institute of Science and Technology, Av. Carl Friedrich Gauss, 3, 08860 Castelldefels, Spain \\ ${ }^{5}$ Istituto di Fotonica e Nanotecnologie, Consiglio Nazionale delle ricerche, Piazza Leonardo da Vinci 32, 20133 Milano, Italy \\ *Corresponding author: daniele.ancora@polimi.it
}

Fluorescence tomography is a well established methodology able to provide structural and functional information on the measured object. At optical wavelengths, the unpredictable scattering of light is often considered a problem to overcome, rather than a feature to exploit. Advances in disordered photonics, have shed new light on possibilities offered by opaque materials, treating them as auto-correlation lenses able to create images and focus light. In this letter we propose tomography through disorder, introducing a modified Fourierslice theorem, cornerstone of the computed tomography, aiming to reconstruct a three-dimensional fluorescent sample hidden behind an opaque curtain. () 2021 Optical Society of America

http://dx.doi.org/10.1364/OL.385970

Optics in disordered media has become an active field of research in the recent years thanks to advances in light shaping techniques and to a deeper understanding of the scattering process [1]. Imaging [2,3] and focusing [4] through opaque curtains have been achieved, paving the road towards innovative applications. Among others, they enabled the usage of multi-mode fiber bundles [5], on-purpose design of random devices to shape focus with desired features [6], disorderedmetasurface engineering [7] up to establishing quantum-secure cryptography protocol [8]. In all these cases, the speckle statistics plays a fundamental role and can be controlled inducing, for example, speckle-amorphity $[9,10]$, or retaining information about an object obscured behind a diffuser [2]. Imaging based on speckle-correlation is a well established technique imported from astronomy for the observation of bright objects behind turbulent atmosphere [11]. Phase retrieval algorithms play a major role in this case and its exploitation has lead to a number of imaging applications [12,13]. There is a vast literature concerning the retrieval of objects hidden behind a scattering curtain, which obtained remarkable results also in the reconstruction of bi-dimensional amplitude and phase masks [14]. However, recent studies have approached three-dimensional hidden imaging in different ways: resolving sources at different depths accounting for the speckle-magnification effect [15], characterizing the diffuser's point-source response and reconstructing using cross-correlations [16] or solving a large-scale inverse problem [17].

In this letter we address the problem of tomographic reconstruction through disorder. Classical computed tomography (CT) relies on the observation of a sample at different angles (sinogram), reconstructing its three-dimensional distribution by solving an inverse problem [18]. In projection geometries the method mostly relies on the Fourier-slice theorem, a mathematical tool that links the measured projection with a planar section in the Fourier space. So far, CT has been applied probing samples with X-ray [19], optical wavelength [20] and others. At optical wavelength, however, scattering is usually seen as a puzzling business. In the following we present a tomographic method that deals with the light scattered off an opaque glass, based on the inversion of the sample's auto-correlation sinogram via a phase retrieval approach. To this end, we extend the concept behind the Fourier-slice theorem to process autocorrelation measurements, rather than direct projections. As a matter of fact, the auto-correlation can be estimated even if the sample is obscured behind an opaque layer under the socalled memory effect regime [21]. The basic principle behind this effect is that the speckle-pattern generated by a single point source is invariant under translations. If the object lies within

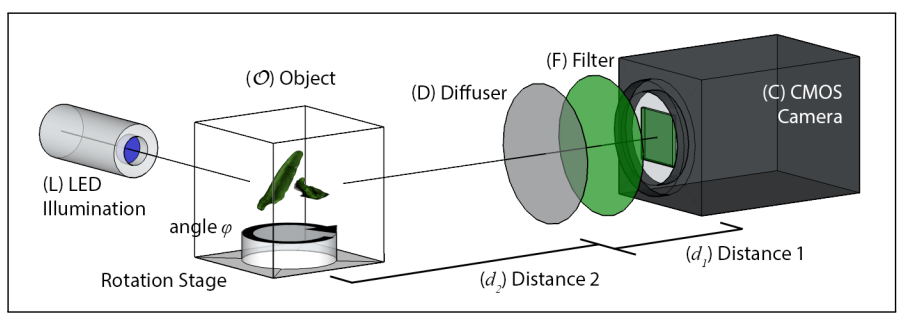

Fig. 1. Sketch of the experimental setup for the imaging of a three-dimensional fluorescent object $\mathcal{O}$ obscured behind an opaque diffuser (D) and a narrow-band filter (F). The fluorescence is excited with a LED (L) and a camera (C) detects the scrambled wave-front. The setup is simple and does not have lenses nor objectives, but rather relies on speckle autocorrelation properties. 


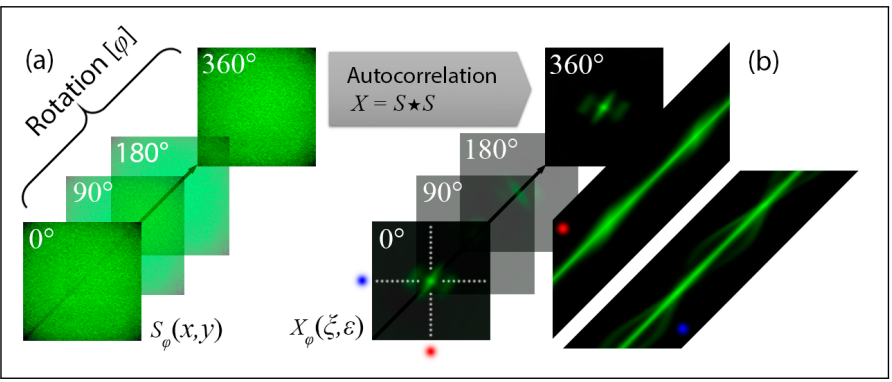

Fig. 2. a) Speckle patterns acquired rotating the object at angle $\varphi . S_{\varphi}$ is corrected by its low-pass envelope to resolve speckle fluctuations. b) Auto-correlation sinogram $X_{\varphi}$ calculated for each $S_{\varphi}$. To visualize the sinogram, we slice the stack $X_{\varphi}$ vertically (red-dot) and horizontally (blue-dot), showing the results in the corresponding dot-labelled images on the right.

the region where the effect holds, this generates -in practice- a random super-position of object's projections that globally share the same auto-correlation with the object itself $[2,3]$. Since we want to reconstruct a three-dimensional object distribution, we assume the object to be smaller than the transverse and axial memory effect region as in [15].

We approach the problem experimentally hiding a fluorescent three-dimensional sample $\mathcal{O}$ behind an opaque diffuser, recording the transmitted intensity field at different angular views as in fluorescent optical projection tomography (OPT) [20]. Rather than forming images with an objective lens, we observe the speckles generated by the light propagating through an unknown ground-glass diffuser. The setup for incoherent speckle tomography is schematically described in Figure 1. A threedimensional fluorescent object $\mathcal{O}$, absorbing at $\lambda_{\text {abs }}=450 \mathrm{~nm}$ and emitting at $\lambda_{\mathrm{em}}=525 \mathrm{~nm}$ (obtained from Microscopy Education FluorRef-Green), is obscured behind an optical diffuser (Thorlabs, ground glass, grit 220). This opaque curtain is placed in front of a camera (Hamamatsu ORCA flash 4.0, pixel dimension of $6.5 \mu \mathrm{m}$ ) at distance $d_{1}=30 \mathrm{~mm}$ and coupled with a bandpass green filter (Brightline, 542/27 nm). We illuminate the sample with a blue LED at $\lambda_{\text {ill }}=450 \pm 18 \mathrm{~nm}$ (Thorlabs, M450LP1) that creates a uniform illumination on the sample using $20 \mathrm{~mm}$ and $50 \mathrm{~mm}$ focal length lenses. The portion of the fluorescence light that propagates through the diffuser reaches the camera after being scrambled by the diffuser itself. In these conditions, the object is not directly recognizable and a speckle pattern is formed on the camera sensor. The 14 bits dynamic range of the camera is adequate to resolve the speckle distribution over a diffused background [3], with a typical exposure time of $1 \mathrm{~s}$. The object is mounted onto a rotation stage (Physik Instrumente M037) at distance $d_{2}=33 \mathrm{~cm}$ from the diffuser and it is rotated for $360^{\circ}$ in steps of $2^{\circ}$. A sequence of speckle patterns is obtained as a function of the rotation angle $\varphi$ with magnification determined by $M=d_{1} / d_{2} \approx 0.09$ [21], thus $1 p x=72.2 \mu \mathrm{m}$. The images of the patterns present low contrast and distortion towards the corners [22] and were corrected by dividing each pattern by its low-pass version (Gaussian kernel with $\sigma=50 p x$ ). Alternatively, Zernike polynomials could be taken into account [22] for a more accurate wave-front correction.

Let us call the speckle-stack produced by the hidden object $\mathcal{O}$ rotated at any given angle $\varphi$ as $S_{\varphi}(x, y)$. In Fig. 2a we show the corrected speckle patterns acquired at angles $\varphi=\left\{0^{\circ}, 90^{\circ}, 180^{\circ}, 360^{\circ}\right\}$. Although the patterns are visually different, it is difficult to identify the object rotation. Since the memory effect holds also along the axial direction [15], each pattern recorded is the convolution of the object's projection $O_{\varphi}$ with the point spread function (PSF) of the diffuser which, in our case, is unknown [2]. Thus, each speckle pattern may be written as $S_{\varphi}=O_{\varphi} * \operatorname{PSF}_{\varphi}$, where $*$ indicates the convolution operator. This is a condition called isoplanatism, where moving the point source through the object does not -substantially- alter the $\operatorname{PSF}_{\varphi}$. Being the $\operatorname{PSF}_{\varphi}$ the random response to a point source located at the object's plane at angle $\varphi$, its auto-correlation will be a sharply peaked function $\operatorname{PSF}_{\varphi} \star \operatorname{PSF}_{\varphi} \approx \delta[2,3]$. This implies that if we take the auto-correlation of $S_{\varphi}$, we have that:

$$
\begin{aligned}
S_{\varphi} \star S_{\varphi} & =\left(O_{\varphi} * \operatorname{PSF}_{\varphi}\right) \star\left(O_{\varphi} * \operatorname{PSF}_{\varphi}\right) \\
& =\left(O_{\varphi} \star O_{\varphi}\right) *\left(\operatorname{PSF}_{\varphi} \star \operatorname{PSF}_{\varphi}\right)=O_{\varphi} \star O_{\varphi} .
\end{aligned}
$$

Here, we assume that $\mathrm{PSF}_{\varphi}$ could also change for different angles as long as its auto-correlation is always close to a $\delta$-function.

This let us estimate the auto-correlation of the object's projection by computing it from the seemingly random signal $S_{\varphi}$. This calculation was done exploiting the ergodic property of the speckle patterns, averaging over different sub-windows of size $128 \times 128 p x$ running within the same camera detection. The number of speckles framed in a single image should be maximized, in order to increase the accuracy of the ensembleaveraged auto-correlation. For this reason, we kept the diffuser at distance $d_{1}=33.5 \mathrm{~mm}$ from the camera sensor. The autocorrelation was calculated using the correlation theorem:

$$
X_{\varphi}(\xi, \varepsilon) \equiv\left\langle S_{\varphi} \star S_{\varphi}\right\rangle=\left\langle\mathcal{F}^{-1}\left\{\left\|\mathcal{F}\left\{S_{\varphi}(x, y)\right\}\right\|^{2}\right\}\right\rangle .
$$

where the angle brackets $\langle\ldots\rangle$ denote the ensemble average of different windows within the same speckle image $S_{\varphi}$. Here, $(\xi, \varepsilon)$ are the shift-coordinates in 2D. In this way, we obtain the auto-correlation sinogram $X_{\varphi}$ that we report in Fig. $2 \mathrm{~b}$ and render in Fig. 3a. Here we can see the resulting auto-correlations $X_{\varphi}$ computed using eq. (2) for each speckle-pattern $S_{\varphi}$ in Fig. 2a. Slicing the stack as a function of the angle, horizontally (blue dot) and vertically (red dot), let us visualize the auto-correlation sinogram where it is possible to observe two rotating side-lobes, whereas no rotation is directly visible in the speckle stack $S_{\varphi}$.

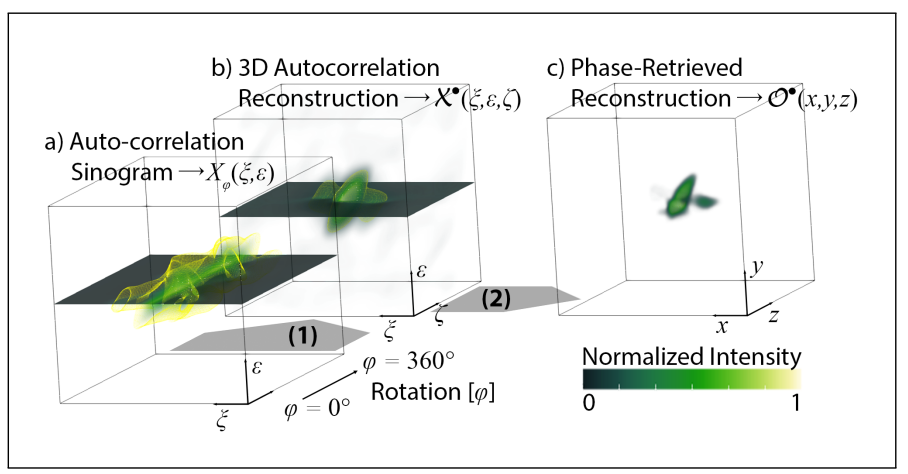

Fig. 3. Volume a): 3D rendering of the auto-correlation sinogram $X_{\varphi}$ previously shown in Fig. 2b. Gray arrow (1): inversion of the $X_{\varphi}$ via SIRT. Volume b): result of the SIRT inversion of $X_{\varphi}$, giving rise to the reconstruction of the auto-correlation $\mathcal{X}^{\bullet}=\mathcal{R}^{-1}\left\{X_{\varphi}\right\}$. Gray arrow (2): 3D phase retrieval algorithm. Volume 3): output of the phase-retrieved fluorescence reconstruction after the recovery of the phase connected to the auto-correlation (operation 2). 
Before discussing the usage of the auto-correlation sinogram $X_{\varphi}$, it is worth introducing the concept of the Radon transform in computed tomography. Given the vastity of the argument, the reader can refer to [18] for more detailed insights on CT. First of all, we define the Radon transform as an operator that projects the function $\mathcal{O}$ (that is the object that we want to measure) along a given set of observation angles $\varphi$ as $\mathcal{R}\{\mathcal{O}\}=O_{\varphi}$. In our case, $\mathrm{O}_{\varphi}$ would be the direct images of the object $\mathcal{O}$ observed at angle $\varphi$. More generally, the $O_{\varphi}$ is the (forward) projection sinogram that can be back-projected to rebuild the object distribution via the inverse Radon transform $\mathcal{R}^{-1}\left\{O_{\varphi}\right\}=\mathcal{O}$. Applying the $\mathcal{R}^{-1}$ implies the solution of an inverse problem, normally addressed via filtered back-projection (FBP) or algebraic reconstruction algorithms [18]. Among the latter, we rely on simultaneous iterative reconstruction technique (SIRT) for any sinogram inversion, however, the study on which is the best inversion method lies well beyond the scope of the present letter

In the following, we will prove that the same principles are applicable in the case of projection's auto-correlation, thus that $\mathcal{R}\{\mathcal{X}\}=X_{\varphi}$ and $\mathcal{R}^{-1}\left\{X_{\varphi}\right\}=\mathcal{X}$. This allow us to estimate the three-dimensional auto-correlation $\mathcal{X}(\xi, \varepsilon, \zeta)$ of the hidden object by inverting the auto-correlation sinogram $X_{\varphi}$ (rendered in Fig. 3a). Here, we denoted with $\zeta$ the shift-coordinate along the $z$-axis. Without loss of generality, we prove this at angle $\varphi=0$, but the concept can be trivially extended to every rotation angle. We call $O_{\varphi=0}=O$ the projection along $z$ of the volumetric object distribution $\mathcal{O}: \mathcal{O}(x, y)=\int \mathcal{O}(x, y, z) d z$. Formally, we have to show that the auto-correlation $\mathcal{X}=\mathcal{O} \star \mathcal{O}$ projected at $\varphi=0$ equals the auto-correlation of the object's projection $X_{\varphi=0}=O \star O$. We do this, by proving the validity of the Fourierslice theorem [18] also in the auto-correlation space. Let us Fourier transform $\mathcal{X}$ and slice it through $k_{z}=0$ :

$$
\begin{aligned}
\left.\mathcal{F}\{\mathcal{X}\}\right|_{k_{z}=0} & =\left\|\mathcal{F}\{\mathcal{O}\}\left(k_{x}, k_{y}, 0\right)\right\|^{2} \\
& =\left\|\int \mathcal{O}(x, y, z) e^{-i 2 \pi\left(x k_{x}+y k_{y}\right)} d x d y d z\right\|^{2} \\
& =\left\|\int \mathcal{O}(x, y) e^{-i 2 \pi\left(x k_{x}+y k_{y}\right)} d x d y\right\|^{2},
\end{aligned}
$$

corresponding to Fourier slice at $\varphi=0$. Here, we notice that we are simply left with:

$$
\left.\mathcal{F}\{\mathcal{X}\}\right|_{k_{z}=0}=\|\mathcal{F}\{O\}\|^{2}=\mathcal{F}\{X\},
$$

which reproduces the Fourier slice theorem for the autocorrelation function. Recalling eq. (2) guarantees that the autocorrelation calculated from the speckle pattern at any angle $X_{\varphi}$ is the projection of the three-dimensional auto-correlation of the hidden object $\mathcal{X}$, thus $\mathcal{R}^{-1}\left\{X_{\varphi}\right\}$ allows its reconstruction. Given eq. (4), we accomplish the sinogram-inversion by using a standard SIRT algorithm (gray arrow labelled with (1) in Fig. 3). This let us obtain the tomographic object's auto-correlation $\mathcal{X} \bullet$ (rendered in Fig. 3b). To roll back to the reconstruction of the object $\mathcal{O}^{\bullet}$ from its auto-correlation we have to solve another inverse problem.

Since we have a valid estimation of the tomographic $\mathcal{X}^{\bullet}$, we can calculate $\mathcal{M}$, the Fourier magnitude of the object, via the Wiener-Kinchin theorem as:

$$
\mathcal{M}\left(k_{x}, k_{y}, k_{z}\right) \equiv\left\|\mathcal{F}\left\{\mathcal{O}^{\bullet}\right\}\right\|=\sqrt{\mathcal{F}\{\mathcal{X} \bullet}
$$

We miss only the phase information to be associated with $\mathcal{M}$ in order to obtain the reconstruction of the hidden object $\mathcal{O}^{\bullet}$.
To do this, we feed this estimation to a phase retrieval (PR) algorithm [11], starting with a random phase $\Phi_{0}\left(k_{x}, k_{y}, k_{z}\right)$. The structure of the auto-correlation is closely related to the structure of the object and could be cleverly used as an additional prior information [23]. PR consists in four-steps iteration in which we:

1. Fourier transform the object's estimation $\mathcal{O}_{i}$ : $\mathcal{F}\left\{\mathcal{O}_{i}\right\}=\left\|\mathcal{F}\left\{\mathcal{O}_{i}\right\}\right\| e^{i \Phi_{i}}$

2. Replace the modulus with the measured one $\mathcal{M}$ : $\mathcal{G}_{i}^{\prime}=\mathcal{M} \frac{\mathcal{F}\left\{\mathcal{O}_{i}\right\}}{\left\|\mathcal{F}\left\{\mathcal{O}_{i}\right\}\right\|}=\mathcal{M} e^{i \Phi_{i}}$

3. Inverse Fourier transform the previous quantity: $\mathcal{O}_{i}^{\prime}=\mathcal{F}^{-1}\left\{\mathcal{G}_{i}^{\prime}\right\}$

4. The object estimation $\mathcal{O}_{i+1}$ is formed by applying different criteria in the region $\gamma$ where the $\mathcal{O}_{i}$ does not satisfy the object constraints (realness and positiveness). We use two different methods in combination, the Hybrid Input-Output (HIO) and the Error Reduction (ER):

$$
\begin{aligned}
& \text { HIO: } \quad \mathcal{O}_{i+1}= \begin{cases}\mathcal{O}_{i}^{\prime} & \text { if }(x, y, z) \notin \gamma \\
\mathcal{O}_{i}-\beta \mathcal{O}_{i}^{\prime} & \text { if }(x, y, z) \in \gamma\end{cases} \\
& \text { ER: } \quad \mathcal{O}_{i+1}= \begin{cases}\mathcal{O}_{i}^{\prime} & \text { if }(x, y, z) \notin \gamma \\
0 & \text { if }(x, y, z) \in \gamma\end{cases}
\end{aligned}
$$

We initially start with the HIO method (typically 1000 iterations, feedback coefficient $\beta=0.8$ ) followed by ER (500 iteration) to lower the reconstruction noise of the output image. The process is schematically represented by the gray arrow (2) in Fig. 3 where we processed a volume of $128^{3}$ voxels. The quality of the reconstruction is assessed by computing the distance $\epsilon_{i}=$ $\|\mathcal{M}-\| \mathcal{F}\left\{\mathcal{O}_{i}\right\}\|\|$. To finalize the result we take the average of 10 best reconstructions with the lowest $\epsilon$ out of 100 trials, forming the final reconstruction $\mathcal{O}^{\bullet}(x, y, z)$ rendered in Fig. 3c.

For comparison, we image the object directly with a telecentric objective lens (Computar TEC-55, 55mm f/2.8 - 32), set to $f / 22$ with a camera exposure time of $0.05 s$. This configuration results into an effective magnification of $M^{\prime}=0.2$ and a pixel size of $1 p x=32.5 \mu \mathrm{m}$. We perform a fluorescence OPT measurement, rotating the object as in the hidden experiment but here acquiring directly the object projections. We centered the axis of rotation of the resulting projection-sinogram by aligning opposite angular projections at $0^{\circ}$ and $180^{\circ}$. To create the ground truth reconstruction, this sinogram was inverted using (SIRT) as in the previous case. This reconstruction is rendered in the box of Fig. 1 and used as reference in Fig. $4 \mathrm{a}$ and 4c.

The three-dimensional object was chosen to have features at different depths, as shown in the ground truth panel in Fig. 4a. In this figure, different depths are color encoded. Compared against Fig. 4a, the reconstruction shown in Fig. 4b correctly retrieved the shape of the hidden object as well as its depthdependent features. To observe a transverse section of the object through the dashed line of Fig. 4a, we slice the ground truth and the retrieved volumes at the same height. These tomographic sections are displayed in Fig. 4c for the ground truth and in Fig. $4 \mathrm{~d}$ for the hidden reconstructions, confirming that features at different depths are correctly resolved. We note that reconstruction converged to higher intensity for the bigger element of the sample. This could be due to the presence of the noise in the calculation of the auto-correlations, adding peaked contribution around the zero-shift region (central part of the auto-correlation) and unbalancing the PR reconstruction. Disentangling the noise contribution from the sinogram $X_{\phi}$ may facilitate quantitative reconstructions, as discussed in [24] in the context of audio signals. The method was tested hiding a different sample behind four diffusers (Thorlabs, grits: 120, 220, 600, 1500) and it always 


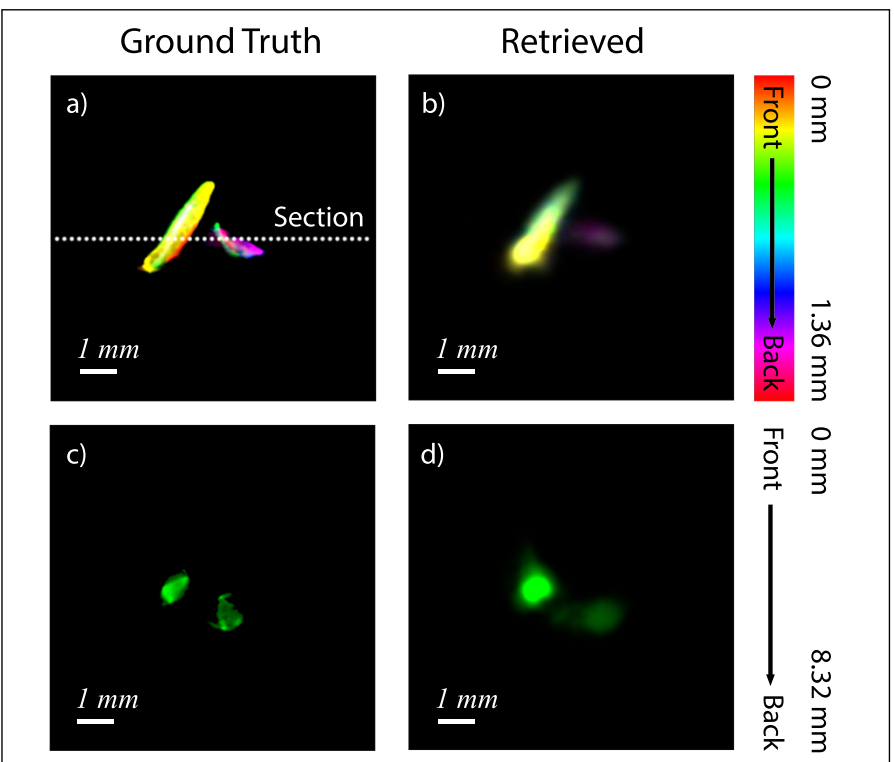

Fig. 4. a), Frontal view (at $\varphi=0^{\circ}$ ) of the ground truth reconstruction of the object $\mathcal{O}$ measured with a normal OPT approach. The location of the fluorescent signal is color-encoded to assess the depth at which the signal is emitted. b) Tomography of the same sample hidden behind the diffuser. The two "legs" are reconstructed at the right depths. c) Tomographic section of the ground truth along the dashed line of panel a). d) Tomographic section of the retrieved volume b) at the same height as in the dashed line of panel a). We can notice the correct location of the two fluorescent objects. The object extends approximately $3.1 \mathrm{~mm}$ along the horizontal axis.

exhibited similar performances (supplementary Fig. S1 [25]).

One of the advantages of our protocol is that it does not require prior knowledge of the position of rotation axis: working in the shift-space avoids misalignment, since the $X_{\varphi}$ always rotates around its center regardless of the object's actual position (Fig. 2b). This guarantees an accurate $\mathcal{X}^{\bullet}$ estimate, independently of the object absolute positioning behind the diffuser. Instead, reconstructing the object's projection angle by angle would turn into independent randomly-positioned images, potentially mirrored against each other. PR algorithms have, in fact, infinite degenerate solutions invariant by spatial translation and axes flip [11]. This would lead to a wrong reconstruction even feeding the retrieval of the projection $O_{\varphi}^{\bullet}$ with the previous $O_{\varphi-1}^{\bullet}$, since the reconstructed sinogram would need an accurate alignment before Radon inversion. Differently to other methods $[16,17]$, we do not require the characterization of the diffuser, perhaps being able to deal with temporal-changes without the need to update the calibration. However, our method may be improved by testing other approaches to carry out inverse Radon transform [26], reconstructing an auto-correlation that better behave with different PR implementations (as, for example, oversampling smoothness [27] or shrink-wrap [23]). So far, we have shown that tomography of three-dimensional hidden object is a concrete possibility, thanks to a modified version of the Fourier-slice theorem that applies to auto-correlations. Unlike conventional OPT approaches, the proposed method has the ability to reconstruct a perfectly aligned volumetric image without the knowledge or calibration of the rotation axis [28] and without any lens system. This may convey phase-retrieval protocols as promising tools in the field of optics, potentially exploitable in other projection-based CT application.

Funding Information. The research has received support from the European Union's Horizon 2020 research and innovation programme under the Marie Sklodowska-Curie project (H2020MSCA-IF-2017, project acronym: HI-PHRET, G.A. 799230) and under H2020 Laserlab Europe V (G.A. 871124).

Acknowledgments. The authors thank Prof. Antonio Pifferi for the scientific and logistic support.

Disclosures. The authors declare no conflicts of interest.

\section{REFERENCES}

1. D. S. Wiersma, Nat. Photonics 7, 188 (2013).

2. J. Bertolotti, E. G. Van Putten, C. Blum, A. Lagendijk, W. L. Vos, and A. P. Mosk, Nature 491, 232 (2012).

3. O. Katz, P. Heidmann, M. Fink, and S. Gigan, Nat. photonics 8, 784 (2014).

4. I. M. Vellekoop, A. Lagendijk, and A. Mosk, Nat. photonics 4, 320 (2010).

5. T. Čižmár and K. Dholakia, Nat. communications 3, 1027 (2012).

6. D. Di Battista, D. Ancora, H. Zhang, K. Lemonaki, E. Marakis, E. Liapis, S. Tzortzakis, and G. Zacharakis, Optica 3, 1237 (2016).

7. M. Jang, Y. Horie, A. Shibukawa, J. Brake, Y. Liu, S. M. Kamali, A. Arbabi, H. Ruan, A. Faraon, and C. Yang, Nat. photonics 12, 84 (2018).

8. S. A. Goorden, M. Horstmann, A. P. Mosk, B. Škorić, and P. W. Pinkse, Optica 1, 421 (2014).

9. D. Di Battista, D. Ancora, M. Leonetti, and G. Zacharakis, Appl. Phys. Lett. 109, 121110 (2016).

10. D. Di Battista, D. Ancora, G. Zacharakis, G. Ruocco, and M. Leonetti, Opt. express 26, 15594 (2018).

11. Y. Shechtman, Y. C. Eldar, O. Cohen, H. N. Chapman, J. Miao, and M. Segev, IEEE signal processing magazine 32, 87 (2015).

12. E. Edrei and G. Scarcelli, Optica 3, 71 (2016).

13. T. Wu, O. Katz, X. Shao, and S. Gigan, Opt. letters 41, 5003 (2016).

14. K. Lee and Y. Park, Nat. communications 7, 1 (2016).

15. Y. Okamoto, R. Horisaki, and J. Tanida, Opt. letters 44, 2526 (2019).

16. S. Mukherjee, A. Vijayakumar, M. Kumar, and J. Rosen, Sci. reports 8 , 1 (2018).

17. N. Antipa, G. Kuo, R. Heckel, B. Mildenhall, E. Bostan, R. Ng, and L. Waller, Optica 5, 1 (2018).

18. A. C. Kak, M. Slaney, and G. Wang, Med. Phys. 29, 107 (2002).

19. J. Hsieh, Computed Tomography: Principles, Design, Artifacts, and Recent Advances, vol. 114 (SPIE Press, 2003).

20. J. Sharpe, Annu. Rev. Biomed. Eng. 6, 209 (2004).

21. I. Freund, M. Rosenbluh, and S. Feng, Phys. Rev. Lett. 61, 2328 (1988).

22. M. Liao, D. Lu, W. He, G. Pedrini, W. Osten, and X. Peng, Appl. Opt. 58, 473 (2019).

23. S. Marchesini, H. He, H. N. Chapman, S. P. Hau-Riege, A. Noy, M. R. Howells, U. Weierstall, and J. C. Spence, Phys. Rev. B 68, 140101 (2003).

24. G. Farahani, EURASIP J. on Audio, Speech, Music. Process. 2017, 13 (2017).

25. D. Ancora, D. Di Battista, A. M. Vidal, S. Avtzi, G. Zacharakis, and A. Bassi, "Doi: 10.6084/m9.figshare.11901927.v1," (2020).

26. A. K. Trull, J. van der Horst, L. J. van Vliet, and J. Kalkman, Appl. optics 57, 1874 (2018).

27. J. A. Rodriguez, R. Xu, C.-C. Chen, Y. Zou, and J. Miao, J. applied crystallography 46, 312 (2013).

28. D. Ancora, D. Di Battista, G. Giasafaki, S. E. Psycharakis, E. Liapis, J. Ripoll, and G. Zacharakis, Sci. reports 7, 11854 (2017). 


\section{FULL REFERENCES}

1. D. S. Wiersma, "Disordered photonics," Nat. Photonics 7, 188 (2013).

2. J. Bertolotti, E. G. Van Putten, C. Blum, A. Lagendijk, W. L. Vos, and A. P. Mosk, "Non-invasive imaging through opaque scattering layers," Nature 491, 232 (2012).

3. O. Katz, P. Heidmann, M. Fink, and S. Gigan, "Non-invasive singleshot imaging through scattering layers and around corners via speckle correlations," Nat. photonics 8, 784 (2014).

4. I. M. Vellekoop, A. Lagendijk, and A. Mosk, "Exploiting disorder for perfect focusing," Nat. photonics 4, 320 (2010).

5. T. Čižmár and K. Dholakia, "Exploiting multimode waveguides for pure fibre-based imaging," Nat. communications 3, 1027 (2012).

6. D. Di Battista, D. Ancora, H. Zhang, K. Lemonaki, E. Marakis, E. Liapis, S. Tzortzakis, and G. Zacharakis, "Tailored light sheets through opaque cylindrical lenses," Optica 3, 1237-1240 (2016).

7. M. Jang, Y. Horie, A. Shibukawa, J. Brake, Y. Liu, S. M. Kamali, A. Arbabi, H. Ruan, A. Faraon, and C. Yang, "Wavefront shaping with disorder-engineered metasurfaces," Nat. photonics 12, 84 (2018).

8. S. A. Goorden, M. Horstmann, A. P. Mosk, B. Škorić, and P. W. Pinkse, "Quantum-secure authentication of a physical unclonable key," Optica 1, 421-424 (2014).

9. D. Di Battista, D. Ancora, M. Leonetti, and G. Zacharakis, "Tailoring non-diffractive beams from amorphous light speckles," Appl. Phys. Lett. 109, 121110 (2016).

10. D. Di Battista, D. Ancora, G. Zacharakis, G. Ruocco, and M. Leonetti, "Hyperuniformity in amorphous speckle patterns," Opt. express 26, 15594-15608 (2018).

11. Y. Shechtman, Y. C. Eldar, O. Cohen, H. N. Chapman, J. Miao, and M. Segev, "Phase retrieval with application to optical imaging: a contemporary overview," IEEE signal processing magazine 32, 87-109 (2015).

12. E. Edrei and G. Scarcelli, "Optical imaging through dynamic turbid media using the fourier-domain shower-curtain effect," Optica 3, 71-74 (2016).

13. T. Wu, O. Katz, X. Shao, and S. Gigan, "Single-shot diffraction-limited imaging through scattering layers via bispectrum analysis," Opt. letters 41, 5003-5006 (2016).

14. K. Lee and Y. Park, "Exploiting the speckle-correlation scattering matrix for a compact reference-free holographic image sensor," Nat. communications 7, 1-7 (2016).

15. Y. Okamoto, R. Horisaki, and J. Tanida, "Noninvasive three-dimensional imaging through scattering media by three-dimensional speckle correlation," Opt. letters 44, 2526-2529 (2019).

16. S. Mukherjee, A. Vijayakumar, M. Kumar, and J. Rosen, "3d imaging through scatterers with interferenceless optical system," Sci. reports $\mathbf{8}$, 1-8 (2018).

17. N. Antipa, G. Kuo, R. Heckel, B. Mildenhall, E. Bostan, R. Ng, and L. Waller, "Diffusercam: lensless single-exposure 3d imaging," Optica 5, 1-9 (2018)

18. A. C. Kak, M. Slaney, and G. Wang, "Principles of computerized tomographic imaging," Med. Phys. 29, 107-107 (2002).

19. J. Hsieh, Computed Tomography: Principles, Design, Artifacts, and Recent Advances, vol. 114 (SPIE Press, 2003).

20. J. Sharpe, "Optical projection tomography," Annu. Rev. Biomed. Eng. 6, 209-228 (2004).

21. I. Freund, M. Rosenbluh, and S. Feng, "Memory effects in propagation of optical waves through disordered media," Phys. Rev. Lett. 61, 23282331 (1988).

22. M. Liao, D. Lu, W. He, G. Pedrini, W. Osten, and X. Peng, "Improving reconstruction of speckle correlation imaging by using a modified phase retrieval algorithm with the number of nonzero-pixels constraint," Appl. Opt. 58, 473-478 (2019).

23. S. Marchesini, H. He, H. N. Chapman, S. P. Hau-Riege, A. Noy, M. R. Howells, U. Weierstall, and J. C. Spence, "X-ray image reconstruction from a diffraction pattern alone," Phys. Rev. B 68, 140101 (2003).

24. G. Farahani, "Autocorrelation-based noise subtraction method with smoothing, overestimation, energy, and cepstral mean and variance normalization for noisy speech recognition," EURASIP J. on Audio,
Speech, Music. Process. 2017, 13 (2017).

25. D. Ancora, D. Di Battista, A. M. Vidal, S. Avtzi, G. Zacharakis, and A. Bassi, "Doi: 10.6084/m9.figshare.11901927.v1," (2020).

26. A. K. Trull, J. van der Horst, L. J. van Vliet, and J. Kalkman, "Comparison of image reconstruction techniques for optical projection tomography," Appl. optics 57, 1874-1882 (2018).

27. J. A. Rodriguez, R. Xu, C.-C. Chen, Y. Zou, and J. Miao, "Oversampling smoothness: an effective algorithm for phase retrieval of noisy diffraction intensities," J. applied crystallography 46, 312-318 (2013).

28. D. Ancora, D. Di Battista, G. Giasafaki, S. E. Psycharakis, E. Liapis, J. Ripoll, and G. Zacharakis, "Phase-retrieved tomography enables mesoscopic imaging of opaque tumor spheroids," Sci. reports 7, 11854 (2017). 